\title{
A029
}

\section{Up/Down Wavefield Decomposition by Sparse} Inversion

\author{
J. van der Neut* (Delft University of Technology) \& F.J. Herrmann \\ (University of British Columbia)
}

\section{SUMMARY}

Expressions have been derived for the decomposition of multi-component seismic recordings into up- and downgoing constituents. However, these expressions contain singularities at critical angles and can be sensitive for noise. By interpreting wavefield decomposition as an inverse problem and imposing constraints on the sparseness of the solution, we arrive at a robust formalism that can be applied to noisy data. The method is demonstrated on synthetic data with multi-component receivers in a horizontal borehole, but can also be applied for different configurations, including OBC and dual-sensor streamers. 


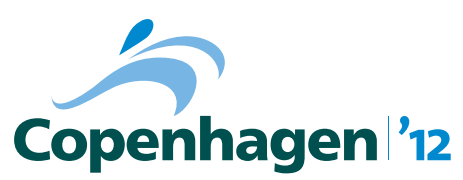

\section{Introduction}

It has been shown that multi-component seismic records can be decomposed into upgoing and downgoing constituents. Up / down decomposition has proved useful for OBC (Ocean Bottom Cable) data (Schalkwijk et al., 1999; Muijs et al., 2007), in dual-sensor streamer technology (Cambois et al., 2009) and in horizontal boreholes (Mehta et al., 2010). If the medium is homogeneous at the receiver array, up / down decomposition can be effectively applied in the frequency-wavenumber (FK) domain. In horizontal boreholes, where the medium parameters are often not homogeneous, decomposition can be applied in the frequency-space (FX) domain by computing pseudo-differential operators. Although these operators can be numerically computed, they tend to be unstable near critical angles and they can be sensitive for noise. Wavefield decomposition can also be interpreted as an inverse problem. To preserve the full frequency content in an optimal sense and remove background noise, we solve this problem with a sparsity promoting inversion scheme in the curvelet domain, which has proven succesfull in a range of geophysical applications (Herrmann et al., 2008). The proposed methodology is demonstrated on synthetic data of a horizontal borehole placed in a heterogeneous medium. The methodology can be extended for elastic media to separate up- and downgoing P- and S-wavefields, for instance for the OBC configuration.

\section{Theory}

Consider measurements of pressure and particle velocity at a horizontal receiver array, expressed by vectors $\mathbf{p}$ and $\mathbf{v}$ in the time-space (TX) domain. The following expression can be derived (Claerbout., 1971; Wapenaar, 1998):

$$
\left(\begin{array}{c}
\mathbf{p} \\
\mathbf{v}
\end{array}\right)=\mathbf{L}\left(\begin{array}{l}
\mathbf{p}^{+} \\
\mathbf{p}^{-}
\end{array}\right)
$$

Here $\mathbf{p}^{+}$and $\mathbf{p}^{-}$represent the down- and upgoing wavefields, respectively, also expressed as vectors in the TX domain. $\mathbf{L}$ is a composition operator that involves forward Fourier transformation, applying pseudo-differential operators (for expressions, see Wapenaar (1998)) and inverse Fourier transformation. Some freedom exist in the scaling of $\mathbf{L}$, depending on what we want the decomposed wavefields $\mathbf{p}^{+}$and $\mathbf{p}^{-}$to represent. In this example we apply power-flux normalization. Although a direct inverse of $\mathbf{L}$ can be numerically computed, this inverse contains singularities near critical angles and can be unstable when applied to noisy data. Instead, we solve equation 1 by sparse inversion in the curvelet domain. Although a feasible approach would be to approximate the composition operator with curvelets, we have another purpose here, namely to impose curvelet-domain sparsity to remove noise. We define a transform $\mathbf{S}=\mathbf{C}_{\mathbf{2}} \otimes \mathbf{W}$, where $\mathbf{C}_{\mathbf{2}}$ is the two-dimensional curvelet transform along the source and receiver coordinates and $\mathbf{W}$ is the discrete wavelet transform. The decomposed data is represented in terms of coefficients $\mathbf{x}^{+}$and $\mathbf{x}^{-}$, according to

$$
\left(\begin{array}{l}
\mathbf{p}^{+} \\
\mathbf{p}^{-}
\end{array}\right)=\left(\begin{array}{cc}
\mathbf{S}^{*} & \mathbf{0} \\
\mathbf{0} & \mathbf{S}^{*}
\end{array}\right)\left(\begin{array}{l}
\mathbf{x}^{+} \\
\mathbf{x}^{-}
\end{array}\right)
$$

Here * represents the adjoint. The representation of seismic data in terms of $\mathbf{x}^{+}$and $\mathbf{x}^{-}$is assumed to be sparse. We minimize the L1-norm of the coefficients with a spectral-gradient projection method (spgl1), while imposing as a constraint that the L2-norm of the residual of equation 1 is bound by noise level $\sigma$ :

$$
\min _{\mathbf{x}^{+}, \mathbf{x}^{-}}\left\|\left(\begin{array}{c}
\mathbf{x}^{+} \\
\mathbf{x}^{-}
\end{array}\right)\right\|_{1} \text { subject to }\left\|\left(\begin{array}{c}
\mathbf{p} \\
\mathbf{v}
\end{array}\right)-\mathbf{L}\left(\begin{array}{cc}
\mathbf{S}^{*} & \mathbf{0} \\
\mathbf{0} & \mathbf{S}^{*}
\end{array}\right)\left(\begin{array}{c}
\mathbf{x}^{+} \\
\mathbf{x}^{-}
\end{array}\right)\right\|_{2} \leq \sigma .
$$




\section{Copenhagen ' 12}

(a)

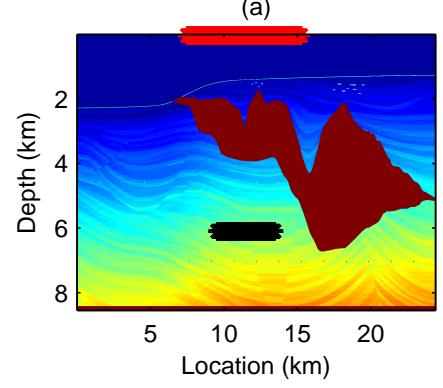

(b)

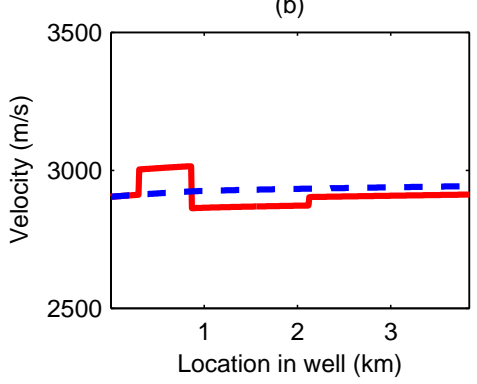

Figure 1 (a) Velocity model with in red the source array and in black the receiver array. (b) True velocity (solid red) and smooth velocity (dashed blue) at the receiver array.

Once the coefficients $\mathbf{x}^{+}$and $\mathbf{x}^{-}$are found, $\mathbf{p}^{+}$and $\mathbf{p}^{-}$can be computed by equation 2 . Following Figueiredo et al. (2008), it is recommended to implement an additional debiasing step to balance the amplitudes after sparse inversion. In this step the residual of problem 1 is further minimized using only the non-zero entries of $\mathbf{x}^{+}$and $\mathbf{x}^{-}$that were found by sparse inversion.

\section{Example}

We test up / down decomposition by sparse inversion on the Sigsbee synthetic model, shown in Figure 1a. Sources and receivers are deployed at the surface and in a horizontal borehole, respectively. In Figure $1 \mathrm{~b}$ we show the true and smoothened velocity profile at the receiver level. For numerical stability, the smoothened velocity profile is used for computation of operator $\mathbf{L}$. In Figures 2 and 3 we show the downhole pressure and particle velocity fields, where Gaussian noise is added with SNR $=5$ (Signal to Noise Ratio, taken with respect to the total wavefield). We focus our attention to the retrieval of the upgoing field, which is weaker than the downgoing field and therefore most challenging to retrieve. For reference we show the upgoing field as retrieved under ideal conditions with no noise in Figure 4. Applying the pseudo-differential operators for decomposition to the noisy input data yields Figure 5. Note that the response contains significantly more noise than Figure 4 and that artifacts can be observed at the critical angle, indicated by the yellow arrows in Figure 5c. These instabilities can be circumvented to some extend by combining up / down decomposition with FK-filtering (van der Neut et al., 2011). The results of such approach are shown in Figure 6. Note from the FK-spectrum (Figure 6c) that the instabilities at critical angles are removed to some extend, but the result is far from ideal and additional artifacts are introduced by the filter. Decomposition by sparse inversion can be applied without FKfilter, see Figure 7. Note that the noise is largely suppressed by the sparseness constraint. We should be warned, however, that some information, such as the reflection indicated by the yellow arrow in Figure 4a, is lost in Figure 7a. In particular applications, such as seismic interferometry (Mehta et al., 2010), such weak signals can contain important information. The aggressiveness of denoising is controlled by noise level $\sigma$. In practice, there is a trade-off between high $\sigma$, for strong denoising, and low $\sigma$, for preserving weak signals.

\section{Conclusion}

The separation of up- and downgoing waves can be interpreted as an inverse problem, which can be solved by sparse inversion. Applying this method to noisy data is robust and does not suffer from singularities at critical angles. The method can be extended to elastic media or be applied with different normalization. 


\section{Copenhagen '12}

(a)

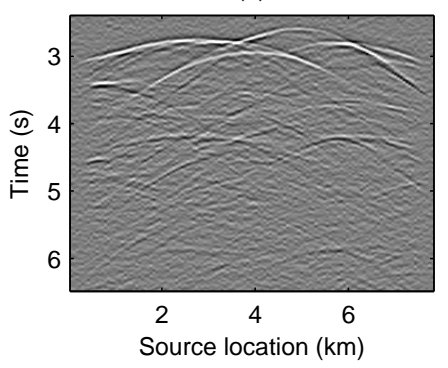

(b)

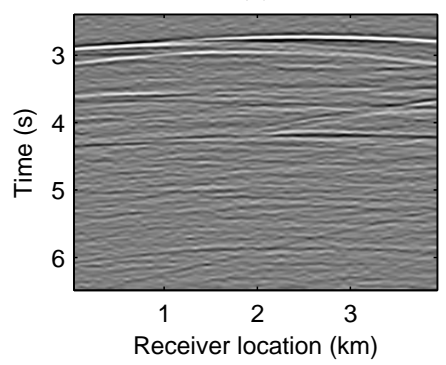

(c)

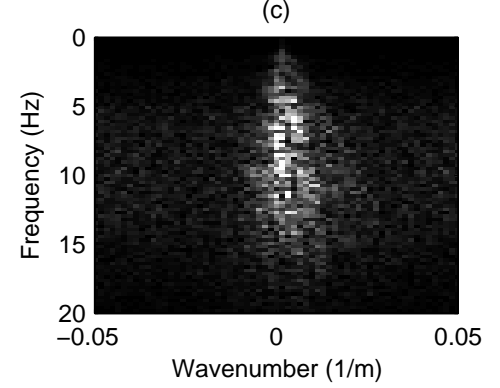

Figure 2 Downhole pressure field with noise: (a) Common receiver gather, (b) Common source gather, (c) FK-spectrum of the common source gather.

(a)

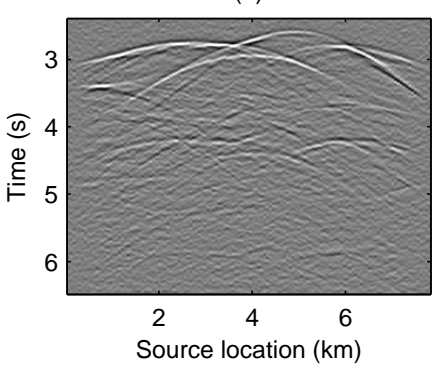

(b)

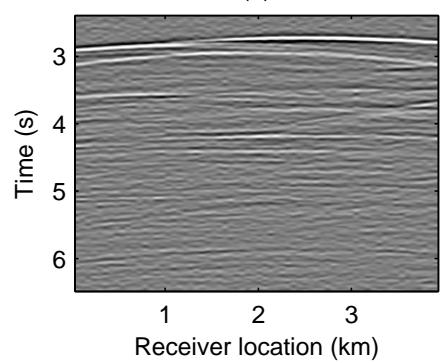

(c)

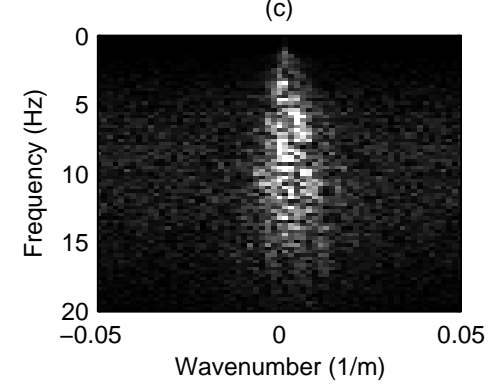

Figure 3 Downhole particle velocity field with noise: (a) Common receiver gather, (b) Common source gather, (c) FK-spectrum of the common source gather.

\section{Acknowledgements}

I would like to thank Tim Lin, Ning Tu, Tristan van Leeuwen, Xiang Li, Ian Hanlon (University of British Columbia) and Kees Wapenaar (Delft University of Technology) for discussions. This work was supported by the Dutch Technology Foundation STW, applied science division of NWO and the Technology Program of the Ministry of Economic Affairs (grant DCB.7913).

\section{References}

Cambois, G., Carlson, D. , Jones, C. , Lesnes, M., Sollner, W. , Tabti, H. and Day, A. [2009] Dual-sensor streamer data, acquisition QC and attenuation of seismic interferences and other noises. 71th EAGE Conference and Exhibition, Expanded Abstracts, X033.

Claerbout, J. F. [1971] Toward a unified theory of reflector mapping. Geophysics, 36, 467-481.

Figueiredo, M. A. T., Nowak, R. D., and Wright, S. J. [2008] Gradient projection for sparse reconstruction: application to compressed sensing and other inverse problems. IEEE Journal of Selected Topics in Signal Processing, 1, 586-597.

Herrmann, F., J., Wang, D., Hennenfent, G., and Moghaddam, P. P. [2008] Curvelet-based seismic data processing: a multiscale and nonlinear approach. Geophysics, 73, A1-A5.

Mehta, K., Kiyashchenko, D., Jorgensen, P., Lopez, J., Ferrandis, J., and Costello, M. [2010] Virtual source method applied to crosswell and horizontal well geometries. The Leading Edge, 29, 712-723.

Muijs, R., Robertsson, J. O. A., and Holliger, K. [2007] Data-driven adaptive decomposition of multicomponent seabed seismic recordings: Application to shallow-water data from the North Sea. Geophysics, 72, 133-142.

Schalkwijk, K. M., Wapenaar, C. P. A., and Verschuur, D. J. [1999] Application of two-step decomposition to multicomponent ocean-bottom data: theory and case study. Journal of Seismic Exploration, 8, 261-278.

Van der Neut, J., Thorbecke, J., Mehta, K., Slob, E., and Wapenaar, K. [2011] Controlled-source interferometric redatuming by crosscorrelation and multidimensional deconvolution in elastic media. Geophysics, 76, SA63SA76.

Wapenaar, K., [1998] Reciprocity properties of one-way propagators. Geophysics, 63, 1795-1798. 


\section{Copenhagen ' 12}

(a)

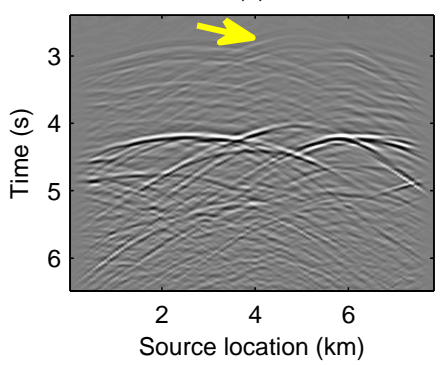

(b)

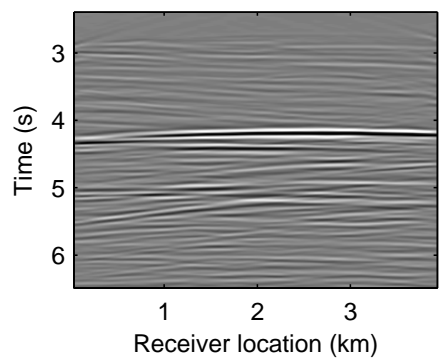

(c)

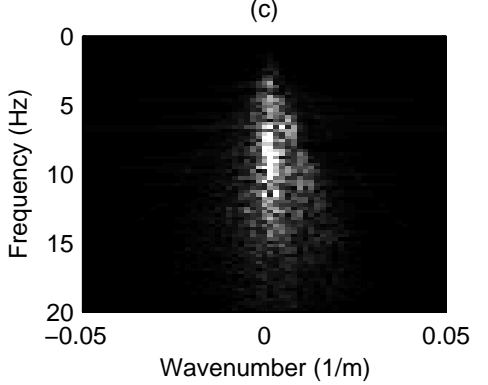

Figure 4 Upgoing field obtained from noise-free data: (a) Common receiver gather, (b) Common source gather, (c) FK-spectrum of the common source gather.

(a)

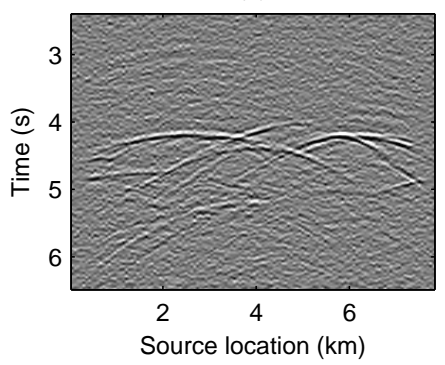

(b)

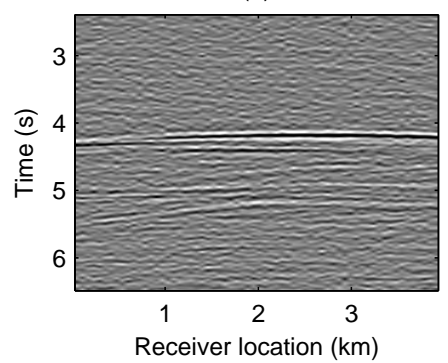

(c)

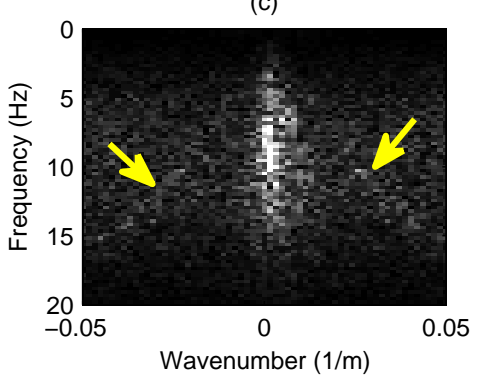

Figure 5 Upgoing field retrieved by direct decomposition from noisy data: (a) Common receiver gather, (b) Common source gather, (c) FK-spectrum of the common source gather.

(a)

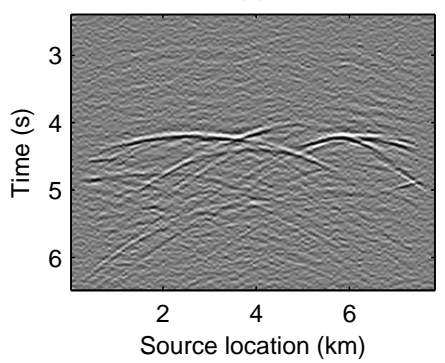

(b)

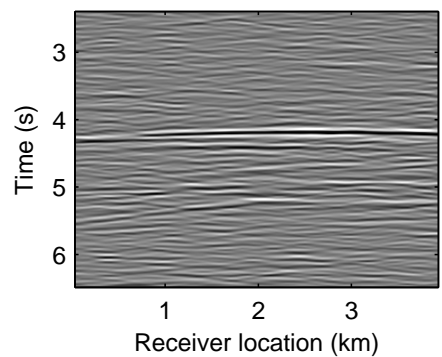

(c)

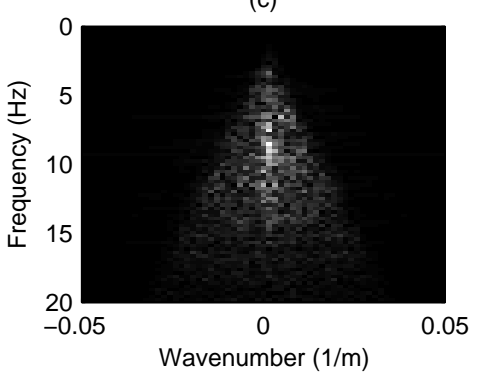

Figure 6 Upgoing field retrieved by direct decomposition with FK-filter from noisy data: (a) Common receiver gather, (b) Common source gather, (c) FK-spectrum of the common source gather.

(a)

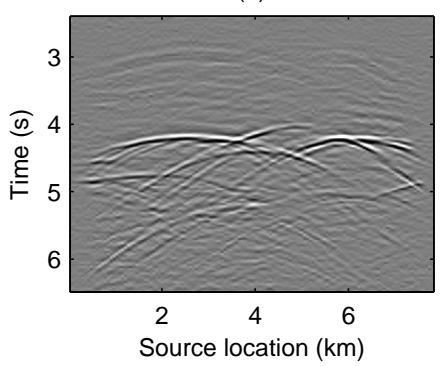

(b)

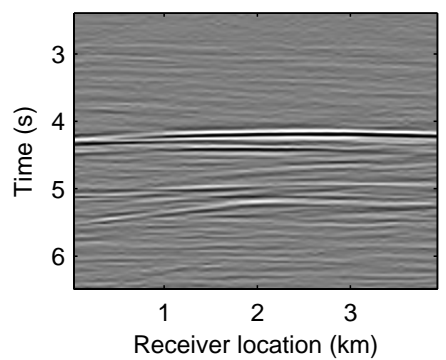

(c)

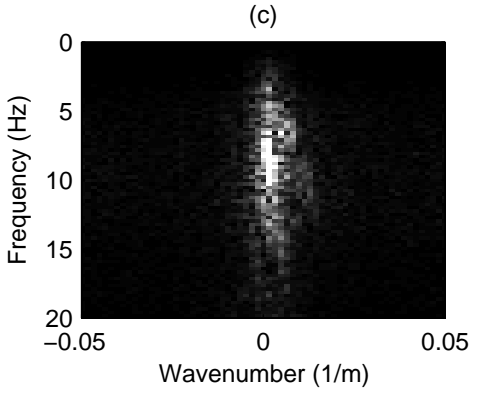

Figure 7 Upgoing field retrieved by sparse inversion from noisy data: (a) Common receiver gather, (b) Common source gather, (c) FK-spectrum of the common source gather. 